\title{
The Vector Vortex Coronagraph: sensitivity to central obscuration, low-order aberrations, chromaticism, and polarization
}

\author{
Dimitri Mawet ${ }^{a}$, Laurent $_{\text {Pueyo }}{ }^{a}$, Dwight Moody ${ }^{a},{\text { John } \operatorname{Krist}^{a} \text {, and Eugene Serabyn }}^{a}$ \\ ${ }^{a}$ Jet Propulsion Laboratory, California Institute of Technology, 4800 Oak Grove Drive, \\ Pasadena, CA 91109, USA
}

\begin{abstract}
The Vector Vortex Coronagraph is a phase-based coronagraph, one of the most efficient in terms of inner working angle, throughput, discovery space, contrast, and simplicity. Using liquid-crystal polymer technology, this new coronagraph has recently been the subject of lab demonstrations in the near-infrared, visible and was also used on sky at the Palomar observatory in the $\mathrm{H}$ and $\mathrm{K}$ bands (1.65 and $2.2 \mu \mathrm{m}$, respectively) to image the brown dwarf companion to HR 7672, and the three extra-solar planets around HR 8799. However, despite these recent successes, the Vector Vortex Coronagraph is, as are most coronagraphs, sensitive to the central obscuration and secondary support structures, low-order aberrations (tip-tilt, focus, etc), bandwidth (chromaticism), and polarization when image-plane wavefront sensing is performed. Here, we consider in detail these sensitivities as a function of the topological charge of the vortex and design features inherent to the manufacturing technology, and show that in practice all of them can be mitigated to meet specific needs.
\end{abstract}

Keywords: High contrast imaging, exoplanets, phase-mask coronagraphy, optical vortex, adaptive optics, wavefront control, small and large space-based telescopes, large and extremely large ground-based telescopes.

\section{INTRODUCTION}

The issue of high contrast imaging of extrasolar planetary systems is dual: the small angular separation between exoplanets and their host stars requires a high angular resolution (100 mas for a 1 AU separation at $10 \mathrm{pc}$ ), and the dynamic range between them is tremendous, from $10^{-3}$ for very close hot giant planets, up to $10^{-10}$ for Earth-like planets. After the first resolved observation of a bound planetary mass object (2M1207 b) was obtained, ${ }^{1}$ several exoplanets (Fomalhaut b, HR 8799 bcd, $\beta$ Pictoris b) have recently been imaged. ${ }^{2-5}$

Direct imaging is clearly a self-consistent and complete characterization method as it provides a straightforward means to constrain orbital parameters and perform spectro-photometric measurements in the most varied orbital configurations. The few exoplanets and the majority of circumstellar disks imaged so far were easy cases, but still required challenging high contrast capabilities. ${ }^{6-8}$ Image dynamic ranges can be increased with a coronagraph, and in a thorough comparison of modern coronagraphs, ${ }^{9}$ two are identified as close to theoretical ideal in terms of "useful throughput": the phase-induced amplitude apodization coronagraph, and the optical vortex phase-mask coronagraph. ${ }^{10-13}$ The optical vortex is truly close to ideal, as it has a small inner working angle (IWA), high throughput, and a completely clear off-axis discovery space. We have therefore been pursuing an especially promising version of the vortex coronagraph, the vector vortex coronagraph (VVC). The vector vortex coronagraph family ${ }^{14}$ provides the basis for a flexible coronagraph solution, adaptable to various telescope configurations. Two near-infrared VVCs (in the $\mathrm{H}$ and Ks band) made out of liquid crystal polymer (LCP) by JDSU are currently available for use with PHARO, the infrared workhorse camera behind the adaptive optics of the 5-m Hale telescope at Palomar. ${ }^{5,15}$ The VVC is currently being considered for various ground- and space-based next-generation high contrast imaging instruments from the $\mathrm{V}$ band to the $\mathrm{N}$ band (see Delacroix et al. 2010, these proceedings).

A high contrast imaging instrument built around the VVC (Fig. 1) responds differently to various degradation factors according to its topological charge and a couple of intrinsic design properties specific to the chosen

Further author information: (Send correspondence to Dimitri Mawet) E-mail: Dimitri.Mawet@jpl.nasa.gov, Telephone: 18183540675

Modern Technologies in Space- and Ground-based Telescopes and Instrumentation, edited by Eli Atad-Ettedgui, Dietrich Lemke, Proc. of SPIE Vol. 7739, 773914 - @ 2010 SPIE · CCC code: 0277-786X/10/\$18 · doi: 10.1117/12.858240 

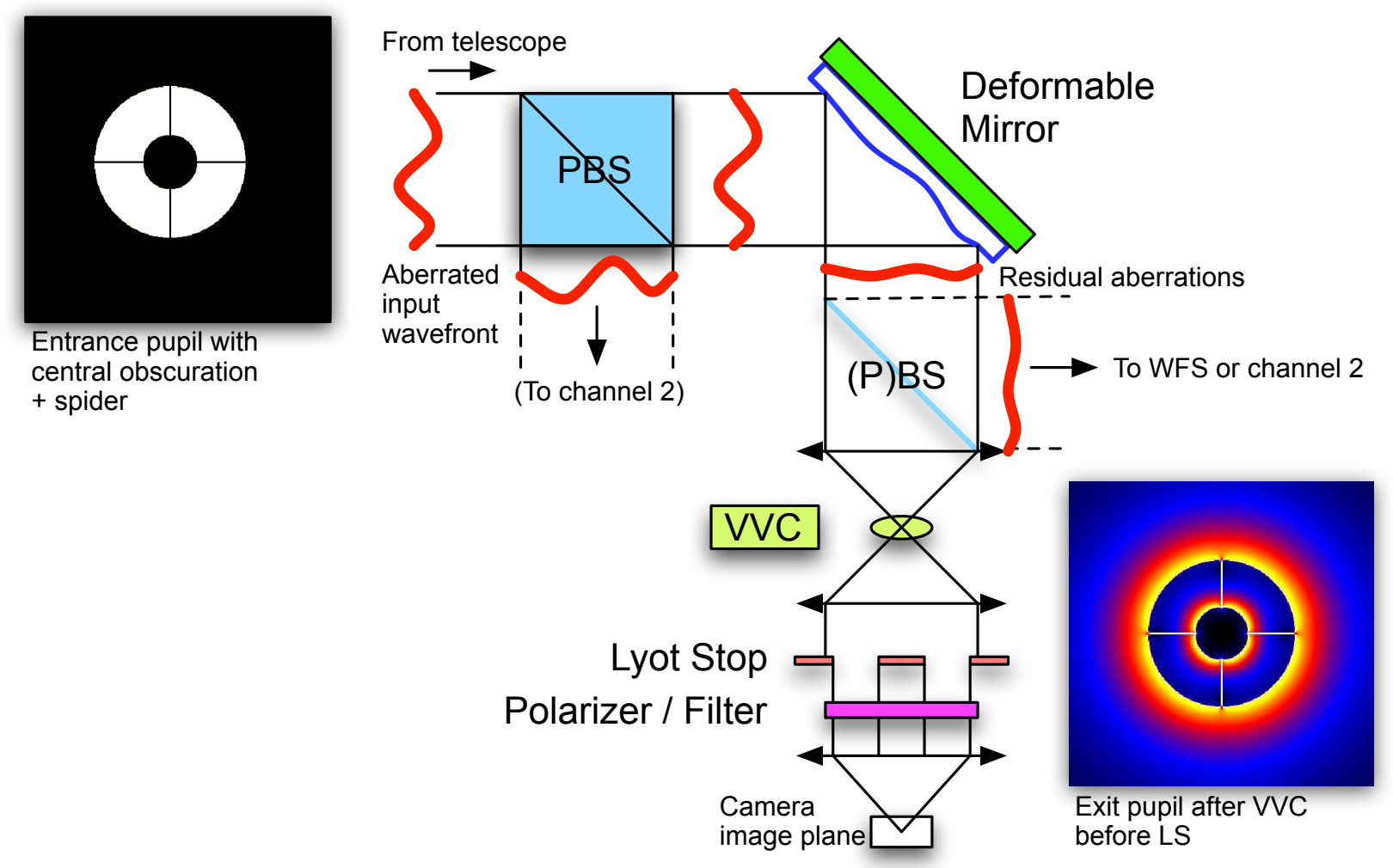

Figure 1. Generic layout of a Vector Vortex Coronagraphic instrument. The telescope may or may not be obscured (see Sect. 3), and will certainly be aberrated (see Sect. 4). Ground-based adaptive optics systems send the light to a dedicated wavefront sensor (WFS) downstream from the deformable mirror (DM) using a beamsplitter (BS). Phase information from the WFS is sent to the DM for partial correction of wavefront aberrations. The residuals (low-order aberrations within the DM passband, and high-order outside the DM passband) are passed through the coronagraph with partial attenuation (see Sect. 4). For pure image-plane sensing schemes (as for space-based coronagraphs), the beamsplitter can be replaced by a polarizing beamsplitter (PBS) that sends the light to a second polarimetric channel (see Sect. 5.2). The beam splitting can be done upstream or downstream of the DM. If done upstream, the second channel needs its own DM, which solves the VVC sensitivity to polarization when image-plane wavefront sensing is chosen (see Sect. 6). LS stands for Lyot stop. Its shape can be more complex than a mere undersized diaphragm to reduce diffraction from the central obscuration seen through the VVC (see exit pupil after VVC inset). The polarizer downstream from the coronagraph can be a potentially useful add-on to the VVC, increasing its working bandwidth by filtering the residual chromatic leakage (see Sect. 5).

technology. In this proceeding, we will consider four kinds of factors that influence the contrast performance of the VVC: the size of the central obscuration if present (Sect. 3), the amplitude of residual low-order aberrations present in the system (Sect. 4), the chromaticism mainly defined by the working bandwidth (Sect. 5), and polarization for image-plane wavefront sensing based systems (Sect. 6).

\section{THE VECTOR VORTEX CORONAGRAPH}

An optical vortex is generated by a phase screw of the form $e^{i \theta}$, with $\theta$ being the azimuthal coordinate. There are two kinds of optical vortex known: the scalar optical vortex, implemented by a structural helix ${ }^{11,13}$ providing a scalar optical phase delay (OPD) which applies to the two orthogonal polarization components of natural light identically, and the vectorial vortex, implemented by a rotationally symmetric halfwave plate (HWP), providing a "geometrical" phase shift ${ }^{16,17}$ that applies opposite phase screws to the two orthogonal circular polarization states (Fig. 2).

The detailed theory of the vector vortex coronagraph is provided elsewhere, ${ }^{10,14}$ and so here we merely 
A. Linear Polarization

B. Circular Polarization

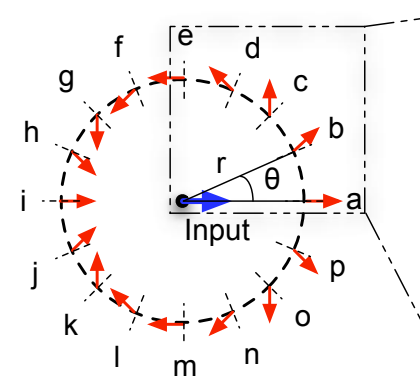

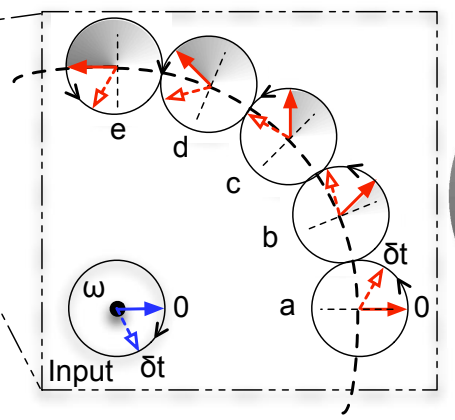

C. Phase ramp: $\phi(\theta)=2 \theta$

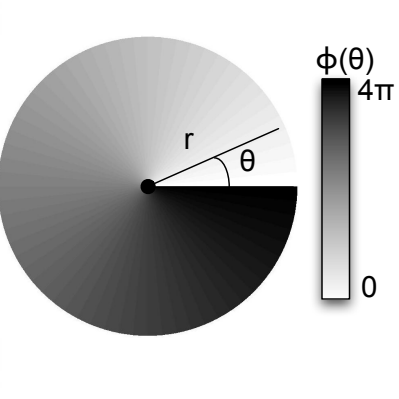

D. FT[e $\left.e^{i 2 \theta} \cdot P S F\right]$

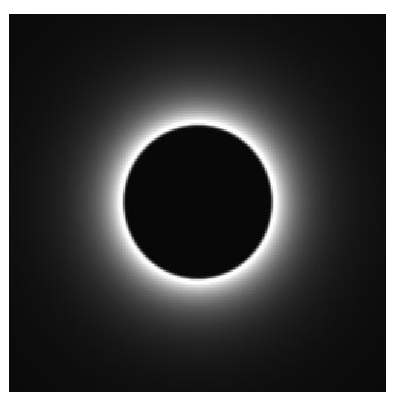

Figure 2. The VVC azimuthal phase ramp. Panel A: rotationally symmetric halfwave plate (HWP) with an optical axis orientation that rotates about the center (dashed lines perpendicular to the circumference). The net effect of a HWP on a linear impinging polarization is to rotate it by $-2 \times \alpha$ where $\alpha$ is the angle between the incoming polarization direction and the fast optical axis. An incoming horizontal polarization (blue arrow) is transformed by the vector vortex so that it spins around its center twice as fast as the azimuthal coordinate $\theta$ (red arrows). Panel B: for circular polarization, the output field rotation is strictly equivalent to a phase delay (the starting angle 0 is rotated; therefore phase shifted). The angle of local rotation of the polarization vector corresponds to a "geometrical" phase: upon a complete rotation about the center of the rotationally symmetric HWP, it has undergone a total $2 \times 2 \pi$ phase ramp, which corresponds to the definition of an optical vortex of topological charge 2 (panel C). Upon propagation from the focal plane to the subsequent pupil plane, the Fourier transform (FT) of the product of the PSF by the azimuthal phase ramp sends the light outside the original pupil area (Panel D).

provide a quick schematic overview. In the vector vortex, for a linearly polarized input field (or for natural light projected onto a linear basis), the rotationally symmetric HWP rotates the polarization vector as in Fig. 2a. The definition of circular polarization is just a linear polarization rotating at the angular frequency $\omega$ (equal to the pulsation of the electromagnetic field), so that a rotation $\phi=2 \theta$ of the polarization vector is strictly equivalent to a phase delay (Fig. 2b). If, at a single plane in space, the polarization vector is instantaneously rotated such as in Fig. 2a, it implies for the circular polarization (Fig. 2b), that it has acquired a geometric phase ramp $e^{i \phi}=e^{i 2 \theta}$ such as represented in Fig. 2c. $\phi$ thus represents both an angle and a phase - hence the term "geometrical" phase. The factor 2 before the azimuthal coordinate in $e^{i 2 \theta}$ is called the "topological charge" $l$. It determines the polarization spin rate, and the subsequent height of the phase ramp after a full $2 \pi$ rotation. In this particular example, we considered a vortex of topological charge 2 , meaning that, upon a complete rotation about the center, it has undergone a total $2 \times 2 \pi$ phase shift.

The final result of the analytical treatment ${ }^{10,14}$ is the "real-world VVC" formula in the circular polarization basis, given in terms of a space-variant Jones matrix (in polar coordinates) as,

$$
J_{v}(r, \theta)=V\left[\begin{array}{cc}
0 & e^{i l \theta} \\
e^{-i l \theta} & 0
\end{array}\right]+L\left[\begin{array}{ll}
1 & 0 \\
0 & 1
\end{array}\right]
$$

where $l$, as defined before, is the vector vortex topological charge, $r$ and $\theta$ the polar azimuthal and radial coordinates, respectively. $J_{v}$ is composed of two main terms. The first term is the pure vortex term of weight $V$, bearing the geometrical phase ramp structure $e^{i l \theta}$. This phase modification is geometrical, i.e. induced only by the space variation of the optical axis orientation across the component. Consequently, it is achromatic by nature. The second term with a weighting coefficient $L$ bears no phase modification and is a chromatic leakage term (see Sect. 5).

Note that this azimuthal phase ramp is generated without a corresponding structural helix. When the pointspread function (PSF) at the focal plane of a telescope is centered on such a vector vortex, for non-zero even values of $l$, the light, upon propagation to a subsequent pupil plane, then appears entirely outside the original pupil area, where it can be rejected by a Lyot stop the same size or slightly smaller than the entrance pupil (Fig. 2d). 

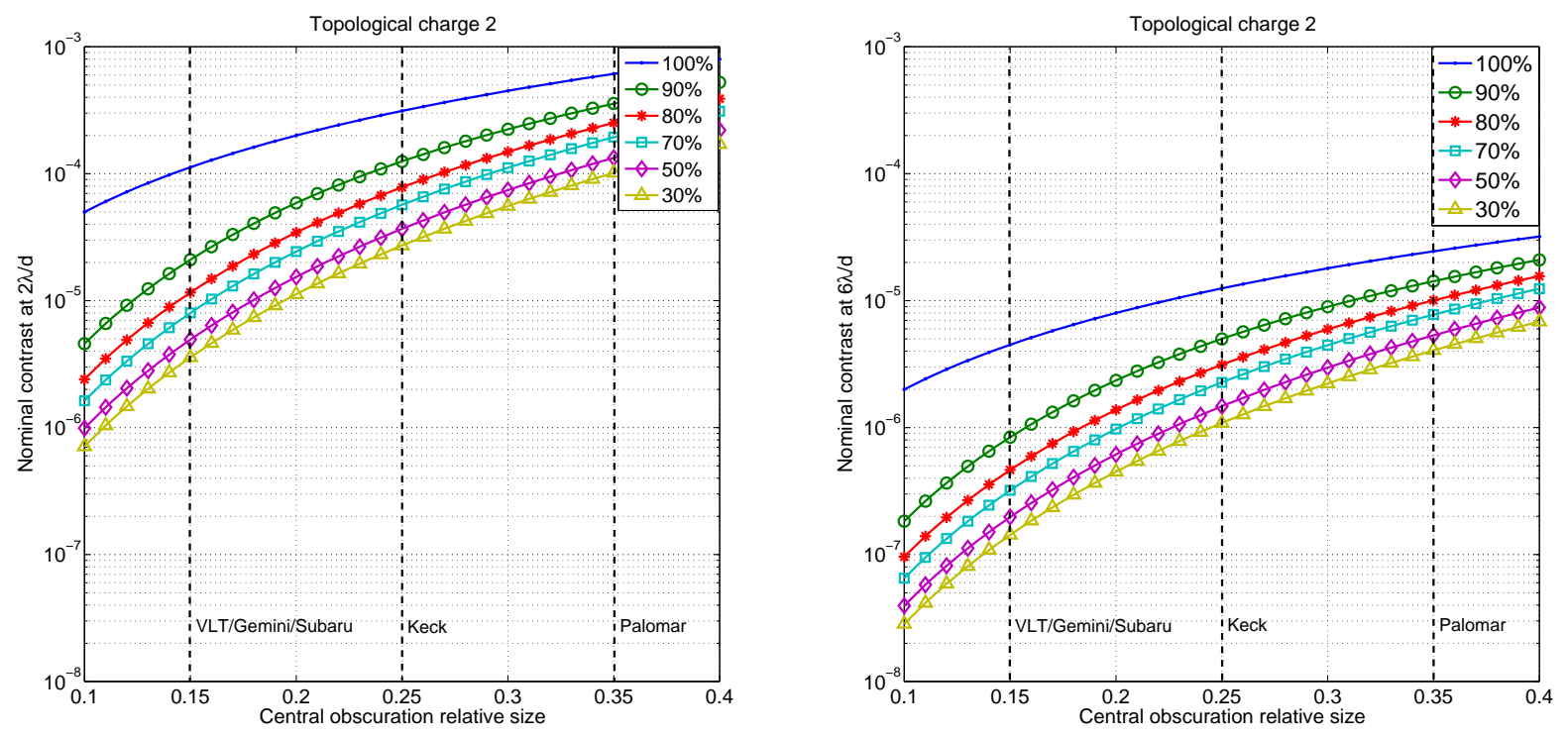

Figure 3. Contrast sensitivity of a topological charge 2 vortex to the central obscuration relative size at the angular separations $2 \lambda / d$ and $6 \lambda / d$ ( $\lambda$ is the observing wavelength and $d$ is the telescope diameter), and for 6 different throughput levels from $100 \%$ down to $30 \%$, respectively. Throughput is traded-off against contrast by oversizing the central obscuration of the Lyot stop. In this computation the outer Lyot stop diameter is equal to the entrance pupil diameter.

\section{SENSITIVITY TO CENTRAL OBSCURATION AND SUPPORT STRUCTURE}

As with the majority of focal plane coronagraphs, the VVC does not escape the fact that the delivered contrast depends on the size of the central obscuration (secondary mirror and any support structures). This adverse effect comes from the simple linear decomposition in amplitude of the obscured pupil into the difference of the same pupil unobscured and a smaller pupil corresponding to the central obscuration, and the action of the VVC on both of them. As described earlier, the VVC redistributes the amplitude from a filled aperture inside out meaning outside the central obscuration as well, i.e. inside the original pupil (see the exit pupil inset in Fig. 1), contaminating it with light in proportion to the area occupied by the central obscuration.

The support structures, and to some extent any gaps from a segmented primary, do not appear to contaminate the pupil outside their own physical extension (see the exit pupil inset in Fig. 1). This is the direct consequence of the edge enhancement property of optical vortices. ${ }^{18}$ Subsequently, an appropriately designed Lyot stop will considerably reduce the scattering due to support structures or gaps in the pupil, without significantly sacrificing throughput (Mawet et al. 2010, in preparation).

\subsection{Sensitivity to central obscuration with a pure VVC}

An unobscured off-axis telescope would ideally be the best configuration for the VVC and almost any coronagraphs, just as the well-corrected subaperture ${ }^{19}$ we used for its first light on the Hale telescope at Palomar observatory. ${ }^{15}$ This experiment was quite unique since for general use ground-based facilities, high-contrast instruments and coronagraphs usually need to adapt to the telescope, and not the inverse. Note that the latter is not true for a dedicated space-based mission, where the whole system can be optimized around a coronagraph or set of coronagraphs to provide optimal performance.

As a matter of fact, the central obscurations of current generation telescopes are typically so small that they are not the primary limitations, which are the residual wavefront errors due to the turbulent atmosphere, even after correction by an adaptive optics system. To some extent, contrast degradation due to the central obscurations can be traded off with throughput, which in general is not an issue for large ground-based telescopes. In Ref. 12, closed-form formulae to estimate the sensitivity to central obscuration as a function of the throughput loss due to the oversizing of the central Lyot stop were presented. The Lyot stop is not a mere diaphragm blocking 

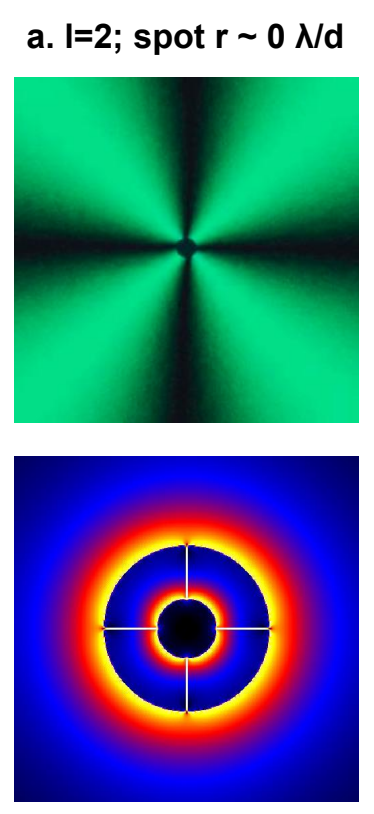
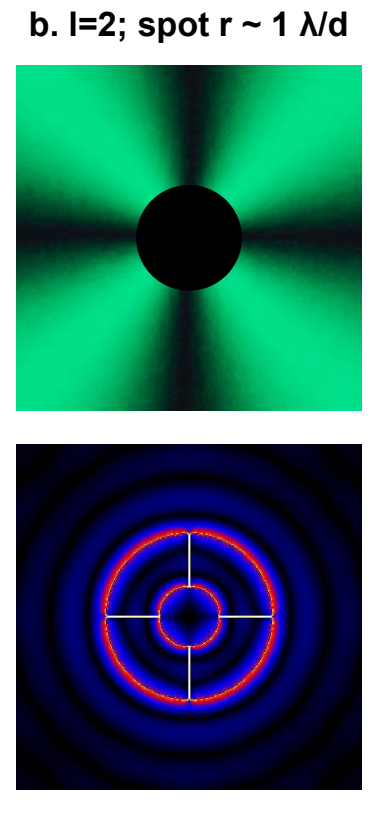
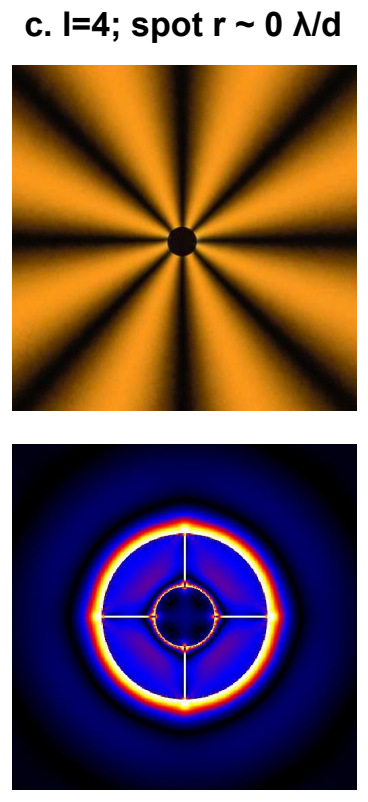

d. I=4; spot $r \sim 1 \mathrm{~N} / \mathrm{d}$
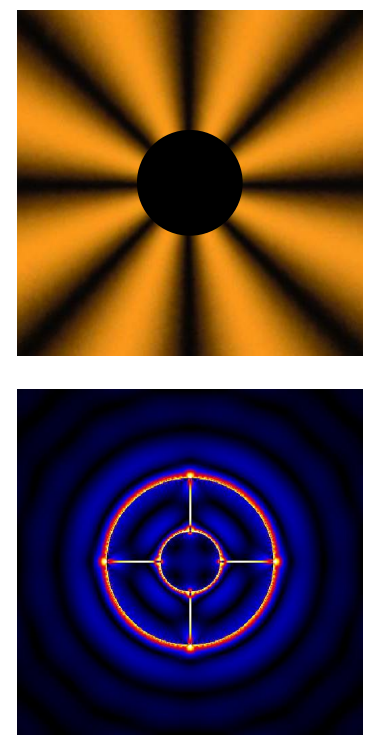

Figure 4. Bottom row: intensity distribution (non-linear but identical stretch for all figures) in the pupil downstream from the VVC for topological charges 2 (panels a and b) and 4 (panels $\mathrm{c}$ and $\mathrm{d}$ ), and for various radii of the central opaque spot $(0 \lambda / d$ and $1 \lambda / d)$. The diffractive effect of the spot can be used to fine tune the pupil diffraction pattern jointly with the Lyot stop with the goal of maximizing contrast and throughput. Note that the spot is always made smaller than the IWA so that the naturally small IWA of the VVC is never penalized by the spot size. The top row shows actual polarimetric microscope images of real devices. One can see the phase modulation only by looking at the sample between crossed polarizers, the device itself being transparent in natural light.

light at the edge of the primary, but also contains an oversized central dark zone oversizing the secondary mirror to block residual diffraction from the secondary mirror.

Figure 3 shows the raw contrast levels obtained for different secondary mirror sizes and throughput trade-offs for a topological charge 2 vortex. The contrast is computed using the analytical formula, ${ }^{12}$ and deriving the contrast at different radial separations. For current generation telescopes (Gemini, Subaru, VLT), the contrast degradation associated with the central obscuration $(<15 \%)$ is very small, and only impacts the raw contrast (before speckle subtraction). The photon noise level associated with such a good raw contrast level, already close to the instrumental limit, is even smaller. These numbers show that a central obscuration is not an obstacle to attaining very good contrast levels with a telescope such as the VLT. For the Palomar telescope, affected by a big secondary mirror (bigger than 30\%), contrast levels are noticeably degraded. For intermediate sizes, close to what it is expected for Extremely Large Telescopes $(\sim 20 \%)$, the contrast loss can be of concern.

\subsection{Sensitivity to central obscuration with a hybrid VVC}

The system can be further optimized, considering the influence of the central opaque spot of the vector vortex made out of LCP (Fig. 4). The spot is a feature specific to the VVC LCP technology. ${ }^{14}$ It is an opaque metal layer $(\mathrm{Al}$, or $\mathrm{Cr})$ with a circular shape creating a high optical density mask 5 to several tens of microns in diameter blocking the VVC center. Note that the spot is always smaller than the IWA of the vortex so that the latter is never penalized by the spot size. Optimizing the size of this spot jointly with the size and shape of the Lyot stop allows reducing the influence of the central obscuration by roughly an order of magnitude with respect to the pure VVC. Figure 4 shows a qualitative view of the effect of the spot size on the diffraction pattern in the pupil downstream the coronagraph. A quantitative idea of the potential gain of this refinement can be seen in Figure 5, where the results of an extensive numerical analysis are presented for a topological charge 2 and 4 vortex, respectively. The conclusion of this computation is that an optimized solution providing deep contrasts $\left(10^{-8}-10^{-7}\right)$ can be found for almost every sensible value of central obscuration ratio possible, and that the 

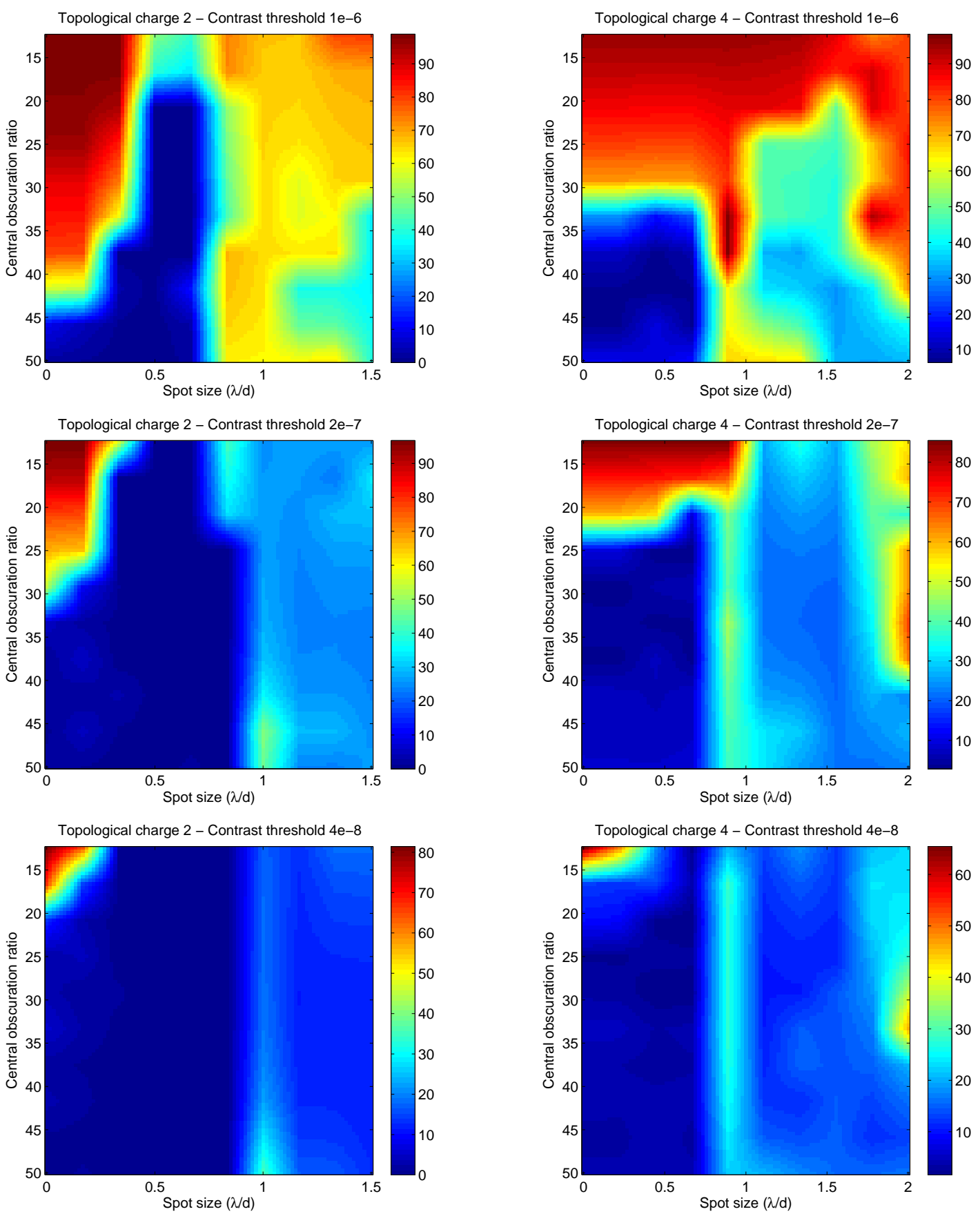

Figure 5. Throughput maps as a function of central obscuration ratio and VVC spot size radius for three distinct contrast thresholds: $<10^{-6},<2 \times 10^{-7},<4 \times 10^{-8}$. The left column is for a topological charge 2 vortex and the right column is for a topological charge 4 vortex. Thanks to the optimal use of the spot, high contrasts $\left(<4 \times 10^{-8}\right)$ can be reached even for large central obscurations if throughput is traded-off.

tradeoff between throughput and contrast is relaxed by roughly an order of magnitude by the careful use of the opaque spot.

Note that radical optical solutions such as beam shaping exist to diminish or totally suppress the influence of the secondary mirror and supports. ${ }^{20-22}$ 


\section{SENSITIVITY TO LOW-ORDER ABERRATIONS AND STELLAR ANGULAR SIZES}
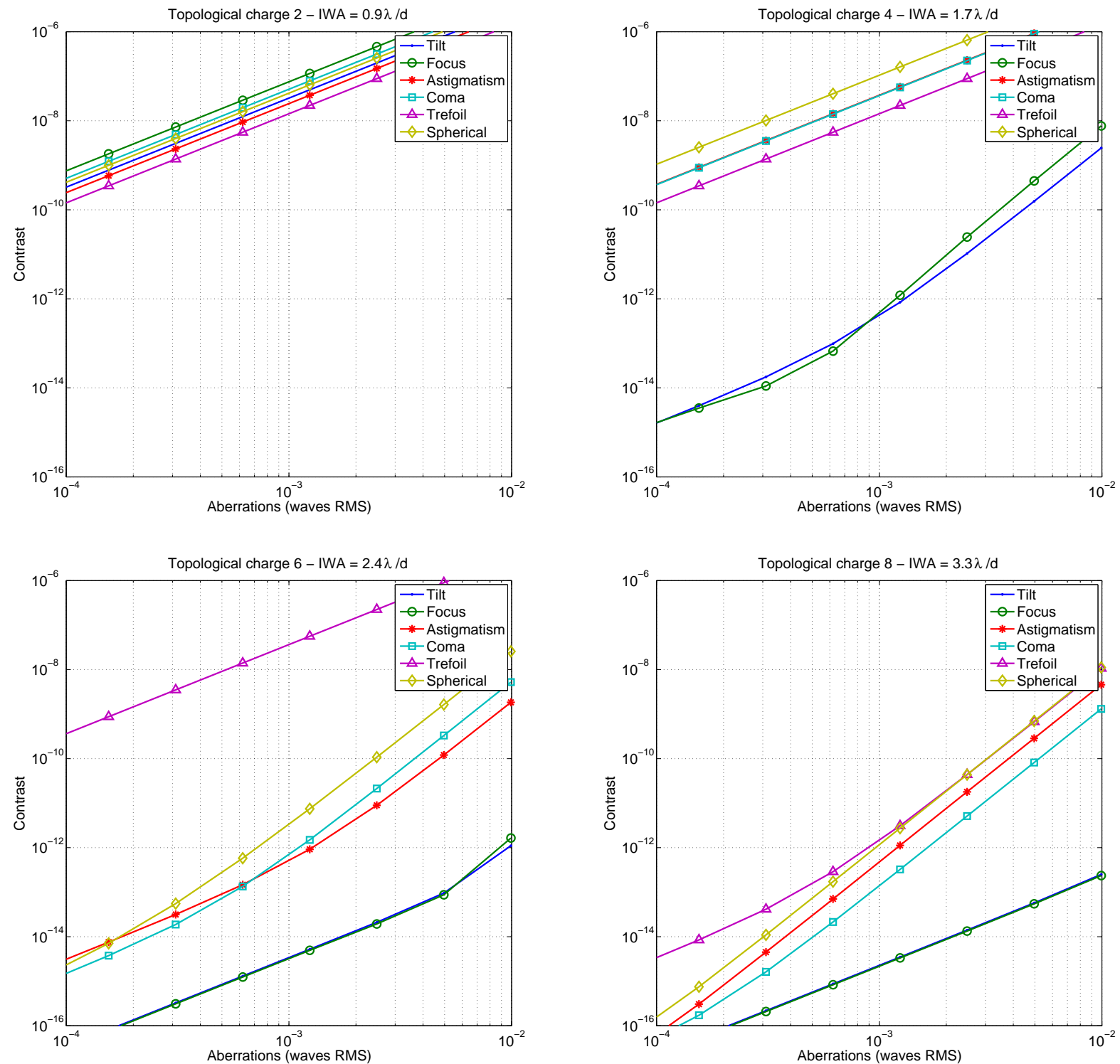

Figure 6. Contrast dependence of topological charge 2, 4, 6, 8 vortices on low-order aberrations between 3 and $4 \lambda / d$. Note the relative sensitivity of charge 2 and the excellent immunity of charge 8 . The best trade-off between IWA and sensitivity to low-order aberrations is for charges 4 and 6.

Table 1. Nominal inner working angle (50\% off-axis throughput) for topological charges 2, 4, 6, 8, and mean contrast leakage between 3 and $4 \lambda / d$ due to stellar angular sizes $s$ (diameter).

\begin{tabular}{cccc}
\hline Topological charge & IWA $(\lambda / d)$ & $s=0.01 \lambda / d$ & $s=0.1 \lambda / d$ \\
\hline 2 & 0.9 & $1 \times 10^{-8}$ & $1 \times 10^{-6}$ \\
4 & 1.7 & $3 \times 10^{-13}$ & $3 \times 10^{-9}$ \\
6 & 2.4 & $8 \times 10^{-18}$ & $8 \times 10^{-12}$ \\
8 & 3.3 & $2 \times 10^{-22}$ & $1 \times 10^{-14}$ \\
\hline
\end{tabular}


The optical vortex coronagraph attenuation sensitivity to low-order aberrations such as tip-tilt $t$ (pointing error) has been shown, numerically, ${ }^{9,10}$ and analytically ${ }^{12}$ to be proportional to $t^{l}, l$ being the topological charge of the vortex. Higher topological charges are therefore less critically sensitive to low-order aberrations. Leakage due to the finite sizes of stars can also be very easily estimated using a model of a uniform stellar disk which is an incoherent superposition of point sources (see Table 1).

Figure 6 show the contrast sensitivity to low order aberrations for Zernike numbers up to 11 (spherical aberration). The calculations were performed using PROPER, ${ }^{23}$ the optical propagation library for IDL. Of interest is the rapid improvement in contrast immunity against aberrations between the topological charge 2 vortex, and the topological charge 8 vortex. The tradeoff between IWA (see Table 1) and the aberration sensitivity suggests that topological charges 4 and 6 represent the best compromises. However, note that lower topological charges do provide access to smaller angles, but at reduced contrast. The VVC coronagraph family thus provides an ensemble of coronagraphic solutions to choose from according to one's needs.

We emphasize that higher topological charges can be easily manufactured with LCP technology. A topological charge 4 vortex has been manufactured by JDSU and tested in the optical on the High Contrast Imaging Testbed (HCIT) at JPL in the framework of a space-based telescope dedicated to exoplanet imaging and characterization. ${ }^{24,25}$ Our manufacturer JDSU has reported the successful manufacturing of topological charge 8 vortices (N. O'Brien, D. Shemo, personal communication). The manufacturing of very high topological charges (up to $l=64$ ) has been reported elsewhere in the literature as well. ${ }^{26}$

\section{SENSITIVITY TO CHROMATICISM}

Chromatic effects are inherent to phase-mask coronagraphs, but their importance greatly depends on the technological approach chosen to render the phase distribution. Well-known techniques to make phase changes achromatic can often be successfully used. ${ }^{27}$ For the vector vortex, chromatic effects appear in the wavelength dependence of the local polarization characteristics of the birefringent medium, i.e. the retardance and the ordinary and extraordinary transmittances. The consequence is that the weighting coefficients $V$ and $L$ in Eq. 1 are wavelength dependent: $V(\lambda)$ and $L(\lambda)$. For the current generation of devices, which is based on a single layer of LCP tuned to a target wavelength, usually picked at the center of the working astronomical filter, the natural dispersion of the LCP material rapidly degrades contrast by contributing quadratically to the leakage on each side of the central wavelength (see top green curve on Fig. 8).

To improve the achromaticity of the vectorial vortex of the single layer LCP design, the first obvious solution is to make the polarization characteristics immune to wavelength changes. This first technique, discussed in Sect. 5.1 consists of using a stack of three LCP layers. Another solution (that can be used jointly with the first one) is to filter the leakage term. This solution, which could potentially bring tremendous improvements with off-the-shelf polarization components, has already been investigated theoretically elsewhere. ${ }^{28}$ Experimental validation of this filtering method with the $\mathrm{VVC}$ will be the subject of future work.

\subsection{3-layer LCP superachromatic VVC}

The 3-layer superachromatic design is currently being investigated for manufacturing. This design again makes use of the geometrical phase, but in a vertical implementation rather than a lateral one. This principle has been known for half a century ${ }^{16}$ and is even commercially available from waveplate vendors. The basic three-layer design consists of identical retarder layers $(1,2,3)$ having nominal retardance values $L_{1}=L_{2}=L_{3}=L_{n o m}=\pi$ radians at a given unique wavelength $\lambda$. The respective optical axes $\alpha_{1}, \alpha_{2}, \alpha_{3}$ of those layers are nominally defined as follows (relative to some arbitrary angle reference): $\alpha_{1}=-30^{\circ}, \alpha_{2}=30^{\circ}+\delta, \alpha_{2}=-30^{\circ}$ (Fig. 7), where $\delta$ is a small modifier angle (i.e. on the order of 0 to a few degrees) that is adjusted to produce the desired bandwidth, at the expense of retardance variation within the band. In practice, the six parameters at each spatial location $\left(L_{1}, L_{2}, L_{3}, \alpha_{1}, \alpha_{2}, \alpha_{3}\right)$ are subject to certain fabrication errors which result in some amount of deviation from the nominal design behavior. The retarder material has also a certain known dispersion of birefringence with wavelength that has to be taken into account in the design.

Designs for a 3 -layer LCP optimized to provide a $10^{-10}$ contrast in a $10 \%$ passband, and another providing a $10^{-9}$ contrast in a $20 \%$ passband were found (Fig. 8) and are being assessed for manufacturing. See Table 2 


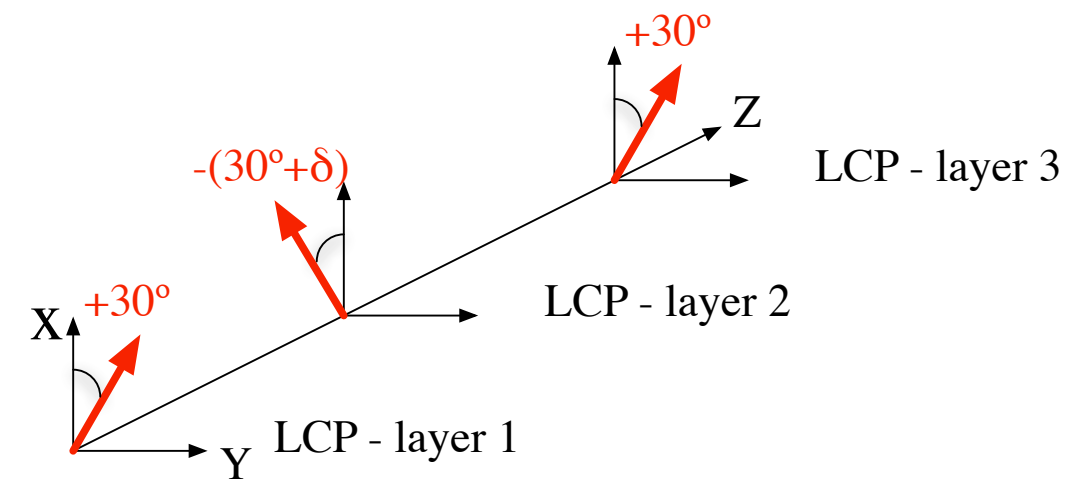

Figure 7. Three-layer LCP design. Optical axis offset between the three identical halfwave layers. Note that each individual layer is only a couple of microns thick (depending on the actual wavelength range of the device). The total thickness of the stack is always very small, on the order of $\sim 10 \mu \mathrm{m}$.
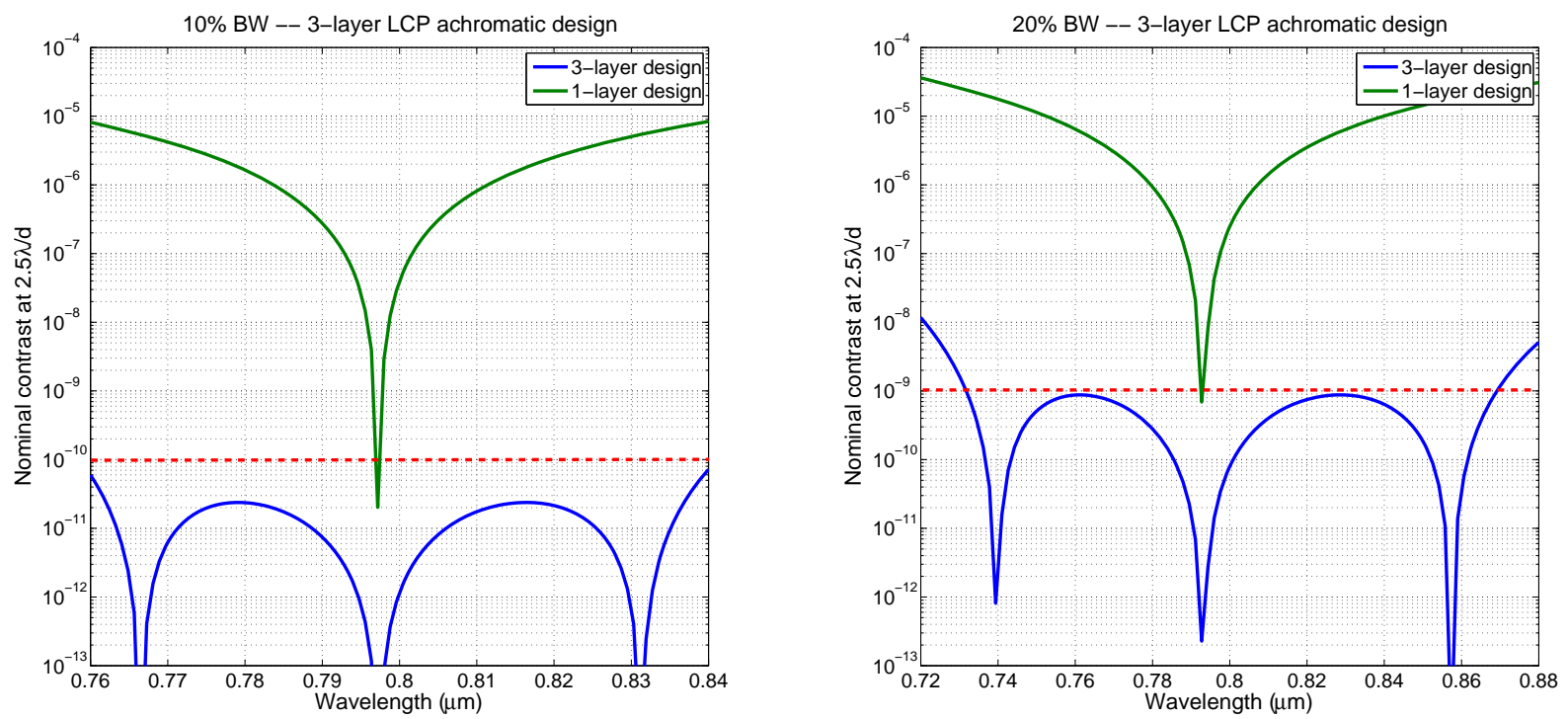

Figure 8. Three-layer LCP designs for $20 \%$ bandwidth (left) and $10 \%$ bandwidth (right). For comparison purposed, the 1-layer design contrast curve is also shown.

for a quantitative estimation of the chromatic leakage contribution to contrast at $2.5 \lambda / d$. For some groundbased instruments, one might want to achromatize a set of passbands simultaneously. A theoretical solution to provide a nominal contrast of $10^{-6}$ at $2.5 \lambda / d$ in three different passbands (Y,J,H) simultaneously is also possible (Table 2).

\subsection{Leakage term filtering and differential polarimetric imaging}

Equation 1, which represents an imperfect chromatic VVC, consists of 2 terms, as already mentioned. A pure geometrical vortex, and a uniform leakage term bearing the chromatic defects. The polarization structure of both terms is orthogonal, which suggests that one could very easily get rid of the leakage term. Indeed, an input left (resp. right) circular polarization state yields a right (resp. left) - circularly polarized vortex term and a left (resp. right) - circularly polarized leakage. An output right (resp. left) - circular analyzer would let the pure vortex term out only, blocking the left (resp. right) - circularly polarized leakage.

To assess the performance gain of such a filtering setup, we use the Jones formalism and realistic assumptions concerning the performance of the circular polarizer/analyser. Indeed, the latter consists of a polarizer with a finite extinction ratio (ER), and a quarterwave plate (QWP) itself affected by the chromaticism of its own 
Table 2. Three-layer LCP designs and performances vs bandwidth in terms of chromatic leakage contribution to the resulting contrast (at $\sim 2.5 \lambda / d$ ), with and without chromatic leakage filtering. These theoretical values are applicable to the entire working wavelength range of the LCP material (from the optical to the near-infrared). Note that as the bandwidth increases, the definition of the IWA becomes flawed because of the PSF size variation with wavelength. Here we used the size of the PSF at the center of the passband.

\begin{tabular}{cccc}
\hline Bandwidth & Modifier angle & Contrast at $\sim 2.5 \lambda / d$ & Leakage filtering \\
\hline $10 \%$ & 0.1 & $\sim 10^{-10}$ & $<10^{-10}$ \\
$20 \%$ & 0.4 & $\sim 10^{-9}$ & $<10^{-10}$ \\
$40 \%$ & 1.0 & $\sim 10^{-7}$ & $\sim 10^{-10}$ \\
$60 \%$ & 2.5 & $\sim 10^{-6}$ & $\sim 10^{-9}$ \\
\hline
\end{tabular}

retardance $\Delta \phi_{Q W P}(\lambda)$. Doing the matrix multiplication leads to a very simple rule of thumb for the filtering attenuation gain $G$ of the orthogonal chromatic leakage:

$$
G \approx\left[\frac{1}{E R}+\Delta \phi_{Q W P}^{2}\right]^{-1}
$$

Assuming off-the-shelf polarizers and waveplate performances, for instance an ER of $10^{4}: 1$ and a QWP retardance accuracy $\Delta \phi_{Q W P}$ of 0.025 radian rms (over the working passband), the relative gain in contrast amounts to $\sim 1400$. This unique property of the VVC would allow us to significantly improve the broadband contrast floor and reduce the requirements on the mask itself. Note that the planetary signal is not affected by the filtering process. A dedicated experiment demonstrating the power of this setup is currently being integrated at JPL (Mawet et al. 2010, in preparation).

Filtering the chromatic leakage imposes the use of polarizing elements upstream of the coronagraph. While adding complexity to the system, it also represents an opportunity to perform an instantaneous polarization measurement AND a real-time calibration of residual speckles. Indeed, polarization can be used both as a tool to facilitate or increase the dynamic range of instruments, and as a scientific gauge to probe physical phenomenon. ${ }^{29,30}$ In fact, starlight that is scattered by gases and aerosol particles in a planetary atmospheres or that is reflected by a planetary surface is generally polarized. The same goes for starlight scattered off of dust in circumstellar disks. The degree and direction of polarization is extremely rich in information. It depends, besides illumination and viewing geometry, on the optical properties of the atmospheric constituents, and the reflection properties of the planetary surface. In particular, the polarization appears to be much more sensitive to the microphysical properties of the atmospheric aerosol particles than the commonly measured spectrum is. Since unresolved starlight (integrated over the disk) is unpolarized, polarization of the signal of interest provides a natural modulation that can be used to improve detectability through demodulation.

Note that the polarization filtering method to achromatize polarization manipulating coronagraphs and diffractive optical elements (DOE) has already been experimentally demonstrated for segmented, discontinuous structures such as the polarization interferometric FQPM ${ }^{31}$ and the Eight-Octant Phase-Mask coronagraph, ${ }^{32}$ as well as for a polychromatic vectorial vortex used as a polarization control/beam shaping DOE. ${ }^{33}$

\section{SENSITIVITY TO POLARIZATION}

Polarization becomes an issue with image-plane wavefront sensing schemes at contrast ratios around $10^{-8}$ and below. Image-plane wavefront sensing is required to thoroughly remove systematic errors in high contrast imaging instruments, or speckles, in order to reach the very high dynamic range necessary to image faint exoplanets. The classical wavefront sensing scheme of Fig. 1, widely used for ground-based adaptive optics systems because of its speed and effectiveness at catching up with the atmospheric turbulence, leaves the aberrations downstream of the wavefront sensor beamsplitter unsensed, so using the final science camera as a sensor is mandatory to remove those so-called non-common path aberrations. Introducing phase diversity either by defocussing an internal source or the camera, either with the deformable mirror (DM), is the most effective way to probe the aberrations down to the science camera. Several schemes have been developed, and the most successful so far in the lab has been the so-called electric field conjugation (EFC) algorithm, ${ }^{34,35}$ which is an evolution of the speckle 
nulling technique. ${ }^{36}$ See also Burruss et al. 2010 (these proceedings), for a detailed description of the modified Gerchberg-Saxton phase retrieval technique used at Palomar to correct non-common path phase aberrations before they hit the VVC (errors downstream from the coronagraph have minimal effects above $10^{-8}$ contrast levels since most of the light is gone at that point).

Because opposite circular polarization states pick up azimuthal phase ramps of opposite signs (see the VVC formula, Eq. 1), phase errors downstream of the vortex mask cannot automatically be corrected in both orthogonal polarizations everywhere in the field simultaneously with a single upstream DM. However, this issue can be completely sidestepped by splitting the two polarizations upstream of the coronagraph (see Fig. 1). This solution not only solves the sensitivity of the VVC to polarization, but also allows the chromatic leakage filtering and polarization simultaneous differential imaging to be performed optimally without throughput loss (see Sect. 5.2). If a single DM is used, sensitivity to polarization can also be mitigated by integrating the electric field information of both polarizations into the wavefront sensing scheme (e.g. EFC) by splitting the 2 orthogonal polarization just before the camera. Limitations will then arise from the level of amplitude errors (including phase-induced amplitude errors) generated downstream from the vortex mask (including amplitude errors generated by the mask itself), and especially by the optics between the vortex and the Lyot stop. If necessary, i.e. if the small number of downstream optics cannot be manufactured with small enough aberrations, those errors can in turn be taken care of by one or more DMs downstream from the coronagraph.

\section{CONCLUSION}

The VVC is a viable coronagraphic solution that has already provided convincing results in the lab and on sky, including the imaging of exoplanets. The VVC does provide access to small angles, has a high throughput, clear $360^{\circ}$ discovery space, and is simple to integrate and use. These characteristics are of prime importance to make the most of a small suborbital or space-based telescope dedicated to extra-solar planetary system imaging and characterization (Serabyn 2010, these proceedings).

In this proceeding, we have studied the contrast sensitivity of the VVC to various degradation factors: the central obscurations found on most ground-based telescopes, and of special concern for the future extremely large telescopes (ELTs) and also of a potential large space-based telescope, low-order aberrations which are critical at the very deep contrasts required to image and characterize mature Jupiter-like planets and Earth-like planets from a space-based platform, chromaticism, and polarization. These issues are clearly identified, but we have shown in this work that practical solutions exist to mitigate all of them to meet specific needs.

\section{ACKNOWLEDGMENTS}

This work was carried out at the Jet Propulsion Laboratory, California Institute of Technology, under contract with the National Aeronautics and Space Administration (NASA).

\section{REFERENCES}

[1] Chauvin, G., Lagrange, A.-M., Dumas, C., Zuckerman, B., Mouillet, D., Song, I., Beuzit, J.-L., and Lowrance, P., "Giant planet companion to 2MASSW J1207334-393254," A $\mathscr{E}$ A 438, L25-L28 (Aug. 2005).

[2] Kalas, P., Graham, J. R., Chiang, E., Fitzgerald, M. P., Clampin, M., Kite, E. S., Stapelfeldt, K., Marois, C., and Krist, J., "Optical Images of an Exosolar Planet 25 Light-Years from Earth," Science 322, 1345(Nov. 2008).

[3] Marois, C., Macintosh, B., Barman, T., Zuckerman, B., Song, I., Patience, J., Lafrenière, D., and Doyon, R., "Direct Imaging of Multiple Planets Orbiting the Star HR 8799," Science 322, 1348- (Nov. 2008).

[4] Lagrange, A.-M., Gratadour, D., Chauvin, G., Fusco, T., Ehrenreich, D., Mouillet, D., Rousset, G., Rouan, D., Allard, F., Gendron, É., Charton, J., Mugnier, L., Rabou, P., Montri, J., and Lacombe, F., "A probable giant planet imaged in the $\beta$ Pictoris disk. VLT/NaCo deep L'-band imaging," $A \& A$ 493, L21-L25 (Jan. 2009).

[5] Serabyn, E., Mawet, D., and Burruss, R., "An image of an exoplanet separated by two diffraction beamwidths from a star," Nature 464, 1018-1020 (Apr. 2010). 
[6] Absil, O. and Mawet, D., "Formation and evolution of planetary systems: the impact of high-angular resolution optical techniques," A\&ARv, 16-+ (Dec. 2009).

[7] Oppenheimer, B. R. and Hinkley, S., "High-Contrast Observations in Optical and Infrared Astronomy," ARAA 47, 253-289 (Sept. 2009).

[8] Duchêne, G., "High-angular resolution imaging of disks and planets," New Astronomy Review 52, 117-144 (June 2008).

[9] Guyon, O., Pluzhnik, E. A., Kuchner, M. J., Collins, B., and Ridgway, S. T., "Theoretical Limits on Extrasolar Terrestrial Planet Detection with Coronagraphs," ApJs 167, 81-99 (Nov. 2006).

[10] Mawet, D., Riaud, P., Absil, O., and Surdej, J., "Annular Groove Phase Mask Coronagraph," ApJ 633, 1191-1200 (Nov. 2005).

[11] Foo, G., Palacios, D. M., and Swartzlander, Jr., G. A., "Optical vortex coronagraph," Optics Letters 30, 3308-3310 (Dec. 2005).

[12] Jenkins, C., "Optical vortex coronagraphs on ground-based telescopes," MNRAS 384, 515-524 (Feb. 2008).

[13] Swartzlander, G. A., Jr., Ford, E. L., Abdul-Malik, R. S., Close, L. M., Peters, M. A., Palacios, D. M., and Wilson, D. W., "Contrast Enhancement of Binary Star System Using an Optical Vortex Coronagraph," Optics Express 16, 10200-10207 (May 2008).

[14] Mawet, D., Serabyn, E., Liewer, K., Hanot, C., McEldowney, S., Shemo, D., and O'Brien, N., "Optical Vectorial Vortex Coronagraphs using Liquid Crystal Polymers: theory, manufacturing and laboratory demonstration," Optics Express 17, 1902-1918 (Feb. 2009).

[15] Mawet, D., Serabyn, E., Liewer, K., Burruss, R., Hickey, J., and Shemo, D., "The Vector Vortex Coronagraph: Laboratory Results and First Light at Palomar Observatory," ApJ 709, 53-57 (Jan. 2010).

[16] Pancharatnam, S. Proc. Indian Acad. Sci. 44, 247 (Jan. 1956).

[17] Berry, M., "The Adiabatic Phase and Pancharatnam's Phase for Polarized Light," Journal of Modern Optics 34, 1401-1407 (Nov. 1987).

[18] Swartzlander, Jr., G. A., "The optical vortex coronagraph," Journal of Optics A: Pure and Applied Optics 11, 094022-+ (Sept. 2009).

[19] Serabyn, E., Wallace, K., Troy, M., Mennesson, B., Haguenauer, P., Gappinger, R., and Burruss, R., "Extreme Adaptive Optics Imaging with a Clear and Well-Corrected Off-Axis Telescope Subaperture," ApJ 658, 1386-1391 (Apr. 2007).

[20] Murakami, N. and Baba, N., "Pupil-remapping Mirrors for a Four-Quadrant Phase Mask Coronagraph," PASP 117, 295-299 (Mar. 2005).

[21] Abe, L., Murakami, N., Nishikawa, J., and Tamura, M., "Removal of central obscuration and spider arm effects with beam-shaping coronagraphy," A\&A 451, 363-373 (May 2006).

[22] Lozi, J., Martinache, F., and Guyon, O., "Phase-Induced Amplitude Apodization on centrally obscured pupils: design and first laboratory demonstration for the Subaru Telescope pupil," ArXiv e-prints (Mar. 2009).

[23] Krist, J. E., "PROPER: an optical propagation library for IDL," in [Society of Photo-Optical Instrumentation Engineers (SPIE) Conference Series], Presented at the Society of Photo-Optical Instrumentation Engineers (SPIE) Conference 6675 (Sept. 2007).

[24] Trauger, J., Stapelfeldt, K., Traub, W., Henry, C., Krist, J., Mawet, D., Moody, D., Park, P., Pueyo, L., Serabyn, E., Shaklan, S., Guyon, O., Kasdin, J., Spergel, D., Vanderbei, R., Belikov, R., Marcy, G., Brown, R. A., Schneider, J., Woodgate, B., Matthews, G., Egerman, R., Polidan, R., Lillie, C., Ealey, M., and Price, T., "ACCESS: a NASA mission concept study of an Actively Corrected Coronagraph for Exoplanet System Studies," in [Society of Photo-Optical Instrumentation Engineers (SPIE) Conference Series], Society of Photo-Optical Instrumentation Engineers (SPIE) Conference Series 7010 (Aug. 2008).

[25] Mawet, D., Trauger, J. T., Serabyn, E., Moody, Jr., D. C., Liewer, K. M., Krist, J. E., Shemo, D. M., and O'Brien, N. A., "Vector vortex coronagraph: first results in the visible," in [Society of Photo-Optical Instrumentation Engineers (SPIE) Conference Series], Society of Photo-Optical Instrumentation Engineers (SPIE) Conference Series $\mathbf{7 4 4 0}$ (Aug. 2009).

[26] Nersisyan, S. R., Tabiryan, N. V., Steeves, D. M., and Kimball, B. R., "The Promise of Diffractive Waveplates," Optics $\mathscr{E}$ Photonics News 21, 40-+ (Mar. 2010). 
[27] Mawet, D., Riaud, P., Baudrand, J., Baudoz, P., Boccaletti, A., Dupuis, O., and Rouan, D., "The fourquadrant phase-mask coronagraph: white light laboratory results with an achromatic device," $A \& A A 448$, 801-808 (Mar. 2006).

[28] Mawet, D., "Annular Groove Phase Mask: An Achromatic Vortex Coronagraph Intended at Differential Polarimetric Imaging," in [In the Spirit of Bernard Lyot: The Direct Detection of Planets and Circumstellar Disks in the 21st Century], Kalas, P., ed. (June 2007).

[29] Thalmann, C., Schmid, H. M., Boccaletti, A., Mouillet, D., Dohlen, K., Roelfsema, R., Carbillet, M., Gisler, D., Beuzit, J., Feldt, M., Gratton, R., Joos, F., Keller, C. U., Kragt, J., Pragt, J. H., Puget, P., Rigal, F., Snik, F., Waters, R., and Wildi, F., "SPHERE ZIMPOL: overview and performance simulation," in [Society of Photo-Optical Instrumentation Engineers (SPIE) Conference Series], Society of Photo-Optical Instrumentation Engineers (SPIE) Conference Series 7014 (Aug. 2008).

[30] Stam, D. M., de Rooij, W. A., Cornet, G., and Hovenier, J. W., "Integrating polarized light over a planetary disk applied to starlight reflected by extrasolar planets," A $\mathcal{J} A \mathbf{4 5 2}, 669-683$ (June 2006).

[31] Baba, N. and Murakami, N., "A Method to Image Extrasolar Planets with Polarized Light," PASP 115, 1363-1366 (Dec. 2003).

[32] Murakami, N., Uemura, R., Baba, N., Nishikawa, J., Tamura, M., Hashimoto, N., and Abe, L., "An EightOctant Phase-Mask Coronagraph," PASP 120, 1112-1118 (Oct. 2008).

[33] Niv, A., Biener, G., Kleiner, V., and Hasman, E., "Polychromatic vectorial vortex formed by geometric phase elements," Optics Letters 32, 847-849 (Apr. 2007).

[34] Give'on, A., Kern, B., Shaklan, S., Moody, D. C., and Pueyo, L., "Broadband wavefront correction algorithm for high-contrast imaging systems," in [Society of Photo-Optical Instrumentation Engineers (SPIE) Conference Series], Society of Photo-Optical Instrumentation Engineers (SPIE) Conference Series 6691 (Sept. 2007).

[35] Moody, D. C. and Trauger, J. T., "Hybrid Lyot coronagraph masks and wavefront control for improved spectral bandwidth and throughput," in [Society of Photo-Optical Instrumentation Engineers (SPIE) Conference Series], Society of Photo-Optical Instrumentation Engineers (SPIE) Conference Series 6693 (Sept. 2007).

[36] Malbet, F., Yu, J. W., and Shao, M., "High-Dynamic-Range Imaging Using a Deformable Mirror for Space Coronography," PASP 107, 386-+ (Apr. 1995). 